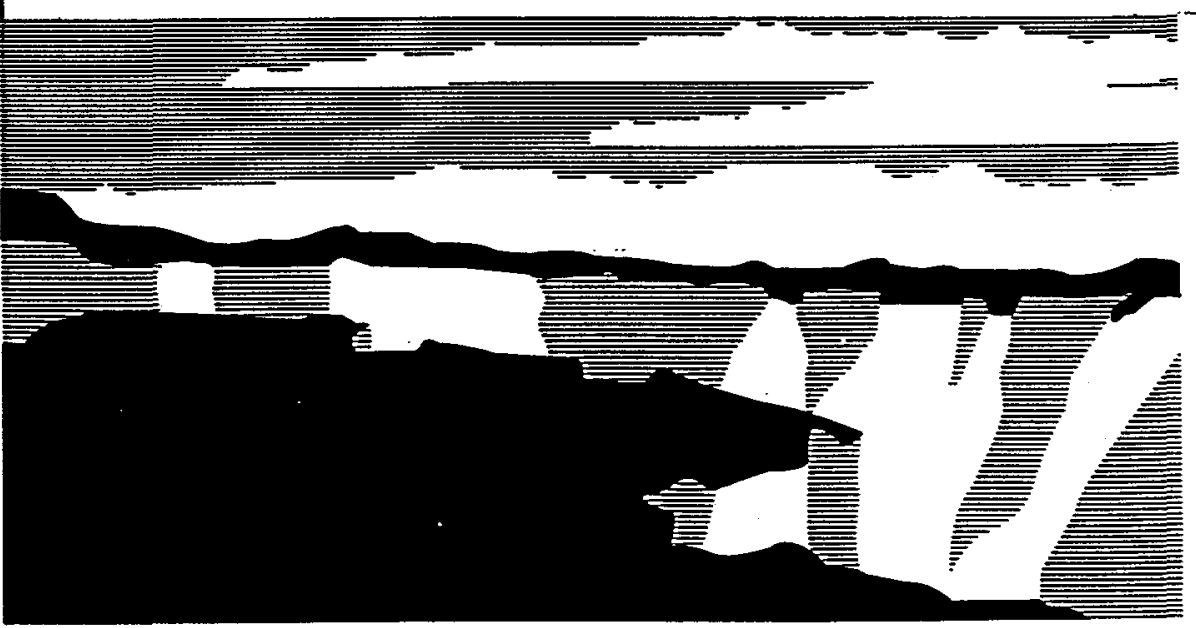

Los Alamos National Laboratory, an affirmative action/equal opportunity employer, is operated by the University of Califomia for the U.S. Department of Energy under contract W-7405-ENG-36. By acceptance of this article, the publisher recognizes that the U.S. Govermment retains a nonexclusive, royalty free license to publish or reproduce the published form of this contribution, or to allow others to do so, for U.S. Govermment purposes. Los Alamos National Laboratory requests that the publisher identify this article as work performed under the auspices of the U.S. Department of Energy. The Los Alamos National Laboratory strongly supports academic freedom and a researcher's right to publish; as an institution, however, the Laboratory does not endorse the viewpolnt of a publication or guarantee its technical correctness. 


\section{DISCLAIMER}

This report was prepared as an account of work sponsored by an agency of the United States Government. Neither the United States Government nor any agency thereof, nor any of their employees, make any warranty, express or implied, or assumes any legal liability or responsibility for the accuracy, completeness, or usefulness of any information, apparatus, product, or process disclosed, or represents that its use would not infringe privately owned rights. Reference herein to any specific commercial product, process, or service by trade name, trademark, manufacturer, or otherwise does not necessarily constitute or imply its endorsement, recommendation, or favoring by the United States Government or any agency thereof. The views and opinions of authors expressed herein do not necessarily state or reflect those of the United States Government or any agency thereof. 


\section{DISCLAIMER}

Portions of this document may be illegible in electronic image products. Images are produced from the best available original document. 


\title{
Predictability of Minimum Noise in Electron Transport Through a Disordered Semiconductor
}

\author{
G.P. Berman ${ }^{1}$, G.D. Doolen ${ }^{1}$, K.E. Nagaev ${ }^{2}$, and J. Rehacek ${ }^{1}$ \\ 1 Theoretical Division and CNLS, Los Alamos National Laboratory, \\ Los Alamos NM 87545 \\ ${ }^{2}$ Institute of Radio-Engineering and Electronics, Russian Academy of Sciences, \\ Mokhovaya Street, 11, 103907 Moscow, Russia
}

\begin{abstract}
We present an inverse method for electron transport which allows one to minimize the value of shot noise for a given value of the conductance by optimizing the spatial structure of a disordered semiconductor region. The method is based on the Green's function approach which is usually applied to the direct transport problem. A specific experimentally realizable example of a two-dimensional disordered semiconductor is presented which demonstrates the method of minimization of shot noise for insulator, metallic, and transitional conductance regions.
\end{abstract}




\section{Introduction}

To calculate the transport properties of a disordered semiconductor, including shot noise, one must know the exact eigenvectors of the Hamiltonian describing this region, and the two semi-infinite electrodes through which the current is fed into the disordered semiconductor. During the past two decades several approaches were developed to treat this problem. (See, for example, reviews [1]-[3] and references therein.) One of the most efficient ways of treating electron transport through disordered mesoscopic quantum systems is the lattice Green's function approach [3]-[7]. In this approach, the semiconductor is considered as a lattice consisting of discrete sites. The electron dynamics in this lattice is described by a matrix Hamiltonian $\hat{H}$,

$$
\hat{H}=\sum_{n} E_{n}|n\rangle\left\langle n\left|+\sum_{n, m} V_{n, m}\right| n\right\rangle\langle m|,
$$

whose indices are the lattice sites. The diagonal elements of $\hat{H}$ represent the binding energies of an electron at each site. The off-diagonal matrix elements represent inter-site electron hopping. Usually, the Hamiltonian (1) is used for two purposes: (a) for investigating the statistical properties of eigenstates and eigenfunctions in closed disordered systems and (b) for investigating the electron transport in open disordered systems. Discussions on both these directions can be found in the review [1] and in references therein. Recently, the Hamiltonian (1) was considered from a different viewpoint [8]. The authors of [8] addressed the strategy of training quantum systems obeying Hamiltonian (1) to satisfy certain physical properties. This approach can be considered as an inverse method when compared with the standard direct approach. In [8] this approach was applied to a closed disordered quantum system described in terms of a two-dimensional Anderson model described by Hamiltonian (1). In the present paper, we consider the inverse problem for electron transport in a disordered semiconductor quantum system described by Hamiltonian (1). The quantum system we consider is an open system which consists of three parts (see Fig. 1): a disordered semiconductor region described by the Hamiltonian (1), and two infinite metallic electrodes ( $\mathrm{L}$ and $\mathrm{R}$ in Fig. 1). The treatment of the disordered region and the electrodes is based on the tight-binding model. Usually, two important characteristics of the dc electron transport are calculated in these systems: the conductance, $\sigma$, and the shot noise, $P$, which are given by the formulae in [1]-[3],

$$
\begin{gathered}
\sigma=\frac{2 e^{2}}{h} \operatorname{Tr} \mathbf{t t}^{\dagger}, \\
P=4 e|V| \frac{e^{2}}{h} \operatorname{Tr} \mathbf{t t}^{\dagger}\left(\mathbf{1}-\mathbf{t t}^{\dagger}\right),
\end{gathered}
$$

where the electron transport properties are described by the transmission matrix $t$, and $V$ is the voltage drop across the disordered region. The problem investigated in 
this paper is the following: for a given value of conductance (2) (in a relatively narrow range $\Delta \sigma$ ) we seek the configuration of a disordered region that minimizes the value of noise $P$ in (3). To simplify the numerical analysis and at the same time to deal with a system which can be easily realized experimentally, we consider a two-dimensional disordered semiconductor that includes $7 \times 7$ sites (see Fig. 1). In all numerical experiments presented below we use dimensionless parameters. The Fermi energy for the left and the right electrodes and for the disordered region is $E_{F}=0$. The site energies $E_{n}$ take three values: $E_{1}=-E, E_{2}=0$, and $E_{3}=E$ (white, gray, and black bullets in Fig. 1, correspondingly). All matrix elements that connect the neighboring sites (bullets in Fig. 1) are equal: $V_{n, m}=V=1$. Each vertical row in Fig. 1 consists of sites with identical energy levels, $E_{n}=0, \pm E$. So, our model consists of seven vertical rows that may differ randomly by the site energies $E_{n}$. Because the site energies take three different values, the total number of realizations for given values $E_{1}, E_{2}$, and $E_{3}$ is $N=3^{7}=2187$. Each realization corresponds to a given value of dimensionless conductance $\sigma /\left(2 e^{2} / h\right)$ (which we denote as $\sigma$ ) and of dimensionless noise $P /\left[4 e|V|\left(e^{2} / h\right)\right]$ (which we denote as $P$ ). Generally, the values of the conductance vary in the range $0 \leq \sigma \leq 7$. Though not in the strict sense, realizations with small values of $\sigma$ will be referred to as "insulating phase"; the region of relatively large values of $\sigma$ will be referred to as "metallic phase", and the intermediate values of $\sigma$ will be referred to as the "region of metal-insulator transition". In numerical experiments we divide the region of available values of $\sigma$ into equal strips of width $\Delta \sigma=0.1$. The number of strips is $M=\left(\sigma_{\max }-\sigma_{\min }\right) / \Delta \sigma\left(\sigma_{\max }\right.$ and $\sigma_{\min }$ were defined without taking into consideration the isolated points). In each strip we find the realization which minimizes the value of noise $P$. Our results show two characteristic properties of the system under consideration. First, the minimizing realizations represent well-correlated dependences on the "conductance - noise" plane. This allows one to predict the value of minimum noise for a given value of conductance. Second, these dependences exhibit different shapes in the region of weak and strong disorder. Below we will analyze the reason for this difference.

\section{Fumerical Results}

Fig. 2a shows the conductance vs. normalized noise $(\sigma, P / \sigma)$ plot for the full ensemble of $M=3^{7}=2187$ realizations for $E=0.5$. One can see that the points are randomly distributed on the plane. At the same time, most points are far from the "insulating" region $(\sigma \ll 1)$. Since the maximum effective number of channels for our system is seven, the maximum value of conductance is also seven. Consequently, region of conductance $(\sigma \in[2.9 ; 6.5])$ shown in Fig. 2a corresponds to the "metallic" phase. The phase diagram for minimizing realizations for this case is presented in Fig. $2 \mathrm{~b}$. One can see that the points on the plot are well correlated. This suggests that using standard statistical methods, one can predict with some accuracy the "minimum noise" in this case. As the diagonal disorder increases, the points on the conductance 
vs. normalized noise $(\sigma, P / \sigma)$ plot shift to smaller $\sigma$. This behavior is demonstrated in Fig. 3a for $E=1$ and for the full ensemble $\left(M=3^{7}=2187\right)$ of realizations. The corresponding plot for minimizing points is shown in Fig. 3b. As in the previous case, one can see well-correlated behavior of the points on the conductance vs. minimum noise plot. As one increases the diagonal disorder, the qualitative behavior of the conductance vs. normalized noise plot changes. This "crossover behavior" is demonstrated for $E=2$ for the full ensemble $\left(M=3^{7}=2187\right)$ of realizations in Fig. $4 \mathrm{a}$ and for minimizing realizations in Fig. 4b. We would like to highlight three characteristic features of the conductance vs. normalized noise" plot in this case. First, as is seen from Fig. 4 , the whole diagram shifts to the region of relatively small values of $\sigma$. This means that the systems becomes more "insulating". Second, the value of the noise goes to zero at $\sigma \approx 1$. This unusual behavior seen in the conductance vs. normalized noise plot may be explained as follows. As the numerical calculations show, for relatively large values of $E$ (as in Fig. 4) the transmission matrix $\mathbf{t}$ becomes nearly diagonal. For example, for $E=2$ and the structure presented in Fig. 1b, the diagonal components of the transmission matrix $t$ are

$$
\begin{gathered}
t_{11}=0.000108785, \quad t_{22}=0.000104155, \quad t_{33}=0.000162928, \quad t_{44}=0.997614, \\
t_{55}=0.000162928, \quad t_{66}=0.000108785, \quad t_{77}=0.000108785 .
\end{gathered}
$$

The off-diagonal matrix elements are of the order $10^{-17}$ in this case. According to Eqs. (2) and (3), the transmission matrix $t$ of the form (4) leads to the characteristic behavior $P \approx 0$ in the vicinity of $\sigma=1$ in the conductance vs. normalized noise plot in Fig. 4. For the same value of the diagonal disorder $E=2$, Fig. 5 shows the conductance vs. noise plot (in this case, the noise, $P$, is not normalized). Third, as one can see from Figs $4 \mathrm{~b}$ and $5 \mathrm{~b}$, the dependence of minimized noise on conductance is also a smooth curve in this case.

In conclusion, we have shown numerical results illustrating how to minimize of shot noise for given values of conductance in different conduction regimes: "metallic", "transitional", and "insulacing". In all these regimes, the conductance vs. minimum noise plots have reasonably smooth curves. This allows one to make predictions of minimum noise for a given value of a conductance in this system. The crossover behavior observed in the conductance vs. minimum noise plots for relatively large values of the diagonal disorder can be used to decrease the value of noise in these systems. We believe that these dependences can be observed in experiments on twodimensional disordered mesoscopic semiconductors.

We would like to thank D.K. Ferry for useful discussions. K.E.N. thanks the Center for Nonlinear Studies, Los Alamos National Laboratory, for hospitality. This work was supported by the Defense Advanced Research Projects Agency. 


\section{References}

[1] C.W.J. Beenakker, Rev. Mod. Phys., 69, 731 (1997).

[2] Y. Imry, Introduction to Mesoscopic Physics, Oxford University Press, 1997.

[3] D.K. Ferry, S.M. Goodnick, Transport in Nanostructures, Cambridge University Press, 1997.

[4] D.S. Fisher, P.A. Lee, Phys. Rev. B, 23, 6851 (1981).

[5] A.D. Stone, Phys. Rev. Lett., 54, 2692 (1985).

[6] P.A. Lee, D.S. Fisher, Phys. Rev. Lett., 47, 882 (1981).

[7] F. Sols, M. Macucci, U. Ravaioli, K. Hess, J. Appl. Phys., 66, 3892 (1989).

[8] I. Kanter, R. Berkovits, Phys. Rev. B, 57, 49 (1998). 


\section{Figure Captions}

Fig. 1. Two realizations in the region $\sigma=1 \pm 0.1 ; E=2\left(E_{1}=-2, E_{2}=0\right.$, $E_{3}=2$ ); (a) $\sigma=1.00352, P / \sigma=0.00603207$; (b) $\sigma=0.998366, P / \sigma=0.0031368$ (a shot-noise minimizing realization in this region).

$E=2\left(E_{1}=-2, E_{2}=0, E_{3}=2\right) ; P / \sigma=0.0543356$.

Fig. 2. Conductance vs. normalized noise $(\sigma, P / \sigma) ; E=0.5$; (a) the total ensemble of $3^{7}$ realizations; (b) minimizing realizations.

Fig. 3. Conductance vs. normalized noise $(\sigma, P / \sigma) ; E=1$; (a) the total ensemble of $3^{T}$ realizations; (b) minimizing realizations.

Fig. 4. Conductance vs. normalized noise $(\sigma, P / \sigma) ; E=2$; (a) the total ensemble of $3^{7}$ realizations; (b) minimizing realizations.

Fig. 5. Conductance vs. noise $(\sigma, P) ; E=r 2$; (a) the total ensemble of $3^{7}$ realizations; (b) minimizing realizations. 

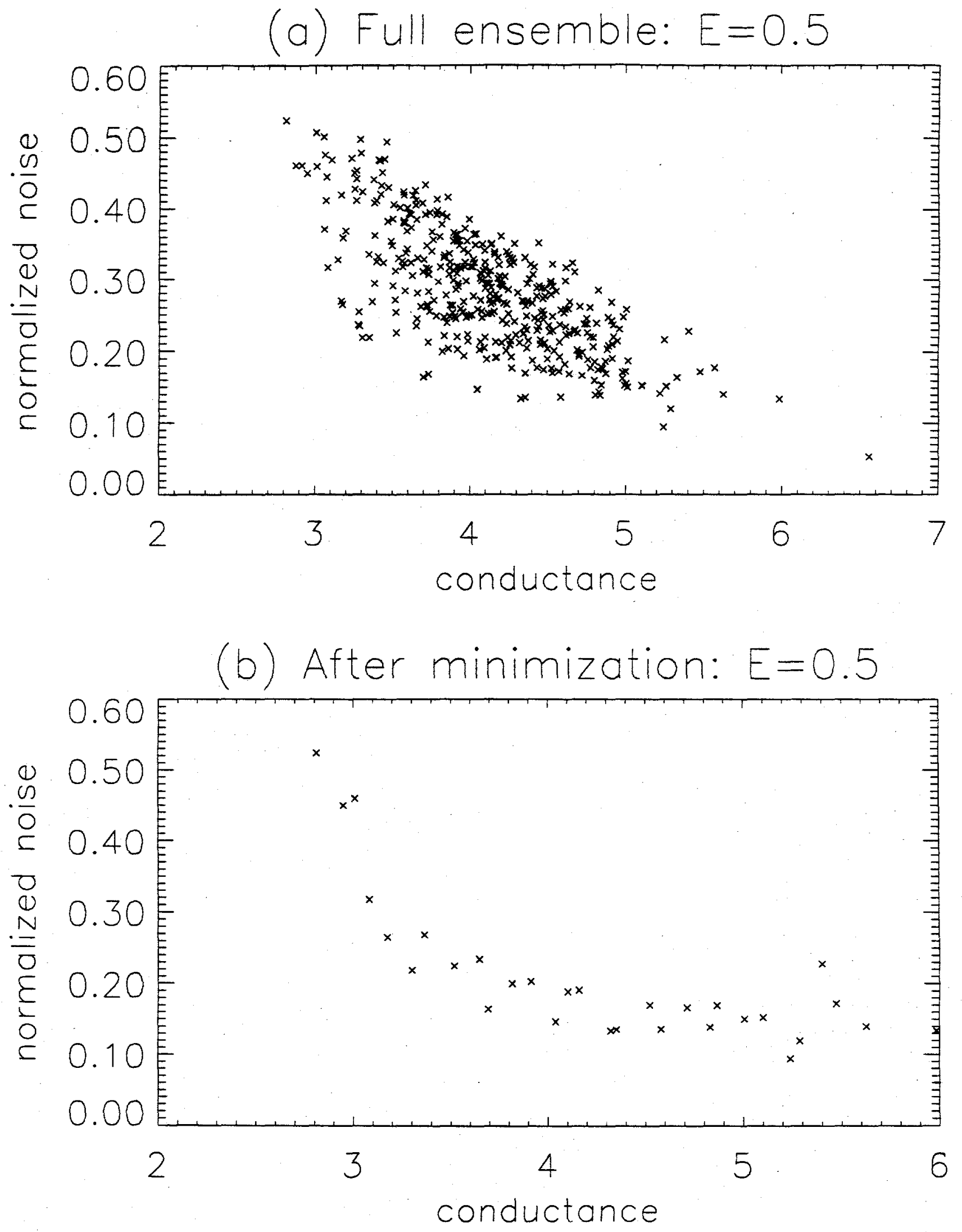


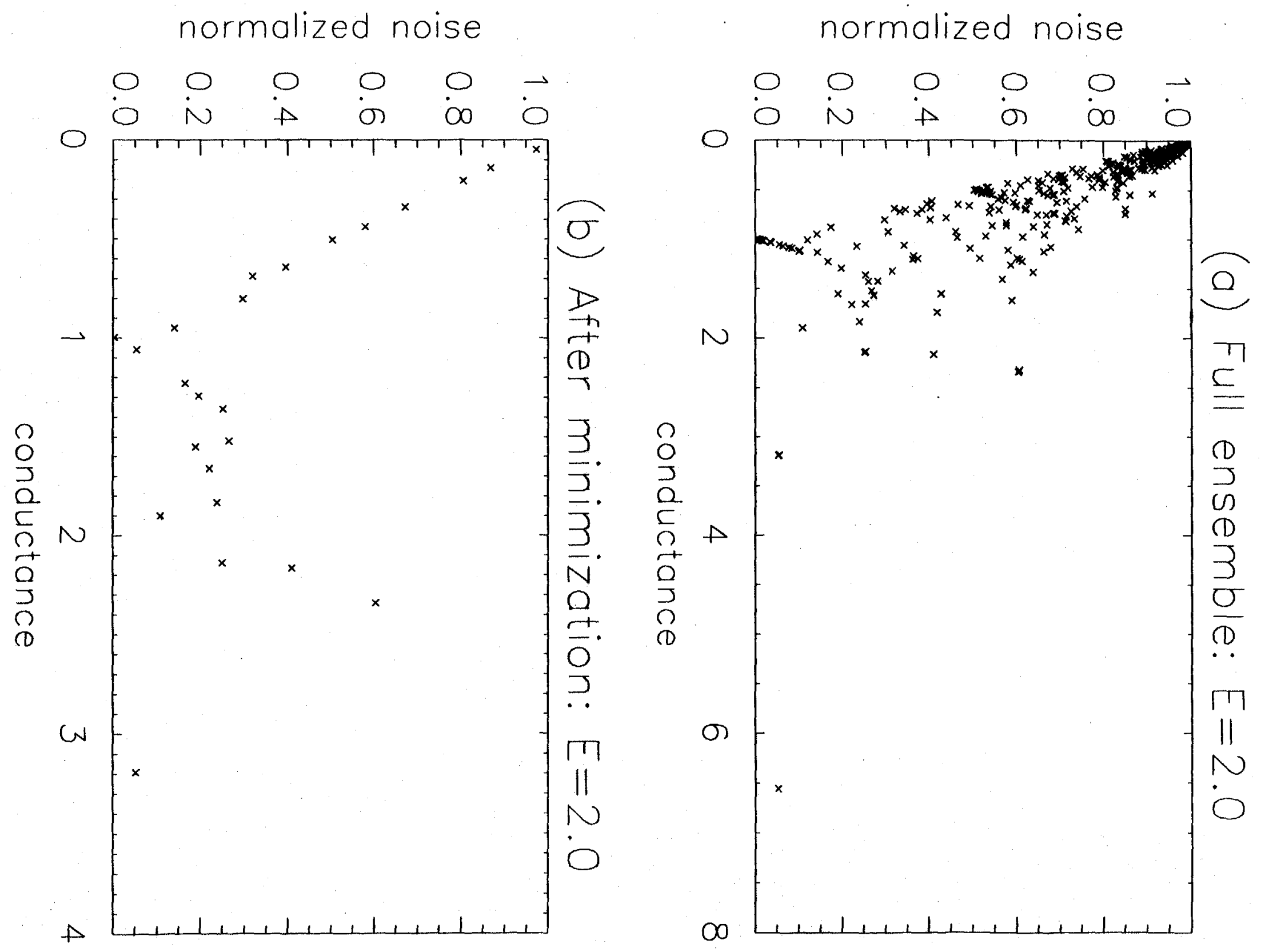